\title{
A FIXED POINT THEOREM FOR MULTIFUNCTIONS IN A LOCALLY CONVEX SPACE
}

\author{
V. M. SEHGAL AND EVELYN MORRISON
}

\begin{abstract}
Let $S$ be a convex subset of a locally convex space $E$ and $K$ a compact subset of $S$. Let $f: S \rightarrow F$ (a topological space) and $g: K \rightarrow F$ be multifunctions. In this paper sufficient conditions are given for the existence of an $x \in K$ such that $f(x) \cap g(x) \neq \varnothing$. The result generalizes a recent theorem of Himmelberg (J. Math. Anal. Appl. 38 (1972), 205-207) and in special cases extends some of the other well-known results.
\end{abstract}

The classical Tychonoff's fixed point theorem [7] has been extended to multifunctions by Browder [1], Fan [2], Glicksberg [4] and several others. Recently, Himmelberg [5] proved the following result, which is a generalization of [2].

TheOREM 1 (HimmelberG). Let $S$ be a nonvoid convex subset of a separated locally convex space $E$. Let $f: S \rightarrow S$ be an u.s.c. multifunction such that $f(x)$ is closed and convex for each $x \in S$, and $f(S)$ is contained in some compact set $K$ of $S$. Then f has a fixed point.

The purpose of this paper is to extend Theorem 1. Recall that a multifunction $f: X \rightarrow Y$ is a point to set function, assigning to each $x \in X$ a nonempty subset $f(x)$ of $Y$. If $A \subseteq X$, then $f(A)=\bigcup\{f(x): x \in A\}$ and if $B \subseteq Y, f^{-1}(B)=\{x \in X: f(x) \cap B \neq \varnothing\}$. It is obvious that if $B=\bigcup B_{i} \subseteq Y$, then $f^{-1}(B)=\bigcup f^{-1}\left(B_{i}\right)$. If $X$ and $Y$ are topological spaces, the multifunction $f: X \rightarrow Y$ is upper semicontinuous iff for each closed set $A \subseteq Y$, $f^{-1}(A)$ is a closed subset of $X$. It is point closed (convex, compact) iff for each $x \in X, f(x)$ is a closed (convex, compact) subset of $Y$.

The proof of the following lemmas can be constructed directly from the definitions.

LEMMA 1. Let $f: X \rightarrow Y$ be a point closed, u.s.c. multifunction on a topological space $X$ into a regular space $Y$. Let $\left\{x_{\alpha}: \alpha \in \Gamma\right\}$ be a net in $X$ which converges to an element $x \in X$. If $y_{\alpha} \in f(x)$ for each $\alpha \in \Gamma$ and if $y_{\alpha} \rightarrow y$, then $y \in f(x)$.

Received by the editors June 20, 1972.

AMS (MOS) subject classifications (1969). Primary 5485, 4785; Secondary 2654, 5465.

Key words and phrases. Multifunctions, upper semicontinuity, fixed point.

(c) American Mathematical Society 1973 
Lemma 2. Let $X, Y$ be topological spaces. If $f: X \rightarrow Y$ is a point compact, u.s.c. multifunction, then the image $f(A)$ of a compact set $A \subseteq X$ is a compact subset of $Y$.

The following result extends Theorem 1 .

THEOREM 2. Let $S$ be a nonempty convex subset of a separated locally convex space $E$ and $K$ a compact subset of $S$. Let $F$ be a regular separated topological space and $f: S \rightarrow F$ be point closed, u.s.c. and $g: K \rightarrow F$ be point compact, u.s.c. multifunctions. If for $x \in S$, (a) $f(x) \cap g(K) \neq \varnothing$, (b) $g^{-1}(f(x))$ is convex, then there is an $x \in K$ such that $f(x) \cap g(x) \neq \varnothing$.

Proof. Let $\mathscr{U}$ be a neighborhood basis of 0 (null element) consisting of a closed, convex and symmetric subset of $E$, and let $U$ be a fixed element of $\mathscr{U}$. Since $K$ is compact, there exists a finite subset $A=\left\{x_{i}: i=1,2, \cdots\right.$, $n\} \subseteq K$ such that $K=\bigcup_{i=1}^{n}\left(\left(x_{i}+U\right) \cap K\right)$. It follows, therefore, that

$$
f^{-1}(g(K)) \subseteq \bigcup_{i=1}^{n} f^{-1}\left(g\left(\left(x_{i}+U\right) \cap K\right)\right) .
$$

Let $S(U)$ be the convex hull of the set $A$. Since $A$ is finite, $S(U)$ is a compact subset of $E$, and by hypothesis (a) and (1) we have

$$
S(U) \subseteq \bigcup_{i=1}^{n} f^{-1}\left(g\left(\left(x_{i}+U\right) \cap K\right)\right)
$$

Define a multifunction $f_{U}: S(U) \rightarrow S(U)$ by

$$
f_{U}(x)=\left(g^{-1}(f(x))+U\right) \cap S(U) .
$$

Note that if $x \in S(U)$, then, by (2), $x_{i} \in g^{-1}(f(x))+U$ for some $i=1,2, \cdots$, $n$ and hence $f_{U}(x) \neq \varnothing$. We show that $f_{U}$ is a point convex, closed u.s.c. multifunction in the relative topology of $S(U)$. The convexity of $f_{U}(x)$, $x \in S(U)$, is obvious by (3). Also, since $f(x)$ is closed, the u.s.c. of $g$ implies that $g^{-1}(f(x))$ is closed and hence compact in $K$. Therefore, $g^{-1}(f(x))+U$ is closed in $E$, that is $f_{U}(x)$ is closed in $S(U)$. To show the u.s.c. of $f_{U}$, let $A$ be a closed (hence compact) set in $S(U)$. Then

$$
\begin{aligned}
f_{U}^{-1}(A) & =\left\{x \in S(U):\left(g^{-1}(f(x))+U\right) \cap S(U) \cap A \neq \varnothing\right\} \\
& =\left\{x \in S(U): x \in f^{-1}(g((A+U) \cap K))\right\} \\
& =f^{-1}(g((A+U) \cap K)) \cap S(U) .
\end{aligned}
$$

Now, since $A+U$ is closed in $E$, it follows by Lemma 2 that $g((A+U) \cap K)$ is compact and therefore $f^{-1}(g((A+U) \cap K))$ is closed in $S$. If

$$
f^{-1}(g((A+U) \cap K))=C \cap S
$$


where $C$ is some closed set in $E$, then (4) implies that $f_{U}^{-1}(A)=$ $C \cap S(U)$ which is a closed set in $S(U)$. Thus, $f_{U}$ is an u.s.c. multifunction. Now $S(U)$ with its relative topology being homeomorphic to the Euclidean $n$-ball (with the usual topology), it follows by Kakutani's theorem [6] that for each $U \in \mathscr{U}$, there exists an element $x_{U} \in S(U)$ such that $x_{U} \in f_{U}\left(x_{U}\right)$, that is

$$
g\left(\left(x_{U}+U\right) \cap K\right) \cap f\left(x_{U}\right) \neq \varnothing .
$$

Let $z_{U} \in U$ and $y_{U} \in g(K)$ such that

$$
y_{U} \in g\left(x_{U}+z_{U}\right) \cap f\left(x_{U}\right) \text {. }
$$

Then the net $\left\{z_{U}: U \in \mathscr{U}\right\} \rightarrow 0$. Also, since $x_{U}+z_{U} \in K$, there is a subnet $\left\{x_{U^{\prime}}+z_{U^{\prime}}: U^{\prime} \in \mathscr{U}^{\prime} \subseteq \mathscr{U}\right\} \rightarrow x \in K$. Now $y_{U^{\prime}} \in g(K)$ implies that a subnet $\left\{y_{U^{\prime \prime}}: U^{\prime \prime} \in \mathscr{U}^{\prime \prime} \subseteq \mathscr{U}^{\prime}\right\} \rightarrow y \in g(K)$. Therefore, restricting to the directed set $\mathscr{U}^{\prime \prime}$, it follows by Lemma 1 and (5) that $y \in g(x) \cap f(x)$. This establishes the proof of Theorem 2 .

COROLlary 1. Let $S$ be a nonempty convex subset of a separated locally convex space $E$, and $K$ a compact subset of $S$. Let $f: S \rightarrow E$ be point closed, u.s.c. and $g: K \rightarrow E$ be point compact, u.s.c. multifunctions. If for $x \in S$, (a) $f(x) \cap g(K) \neq \varnothing$, (b) $g^{-1}(f(x))$ is convex, then there is an $x \in K$ such that $f(x) \cap g(x) \neq \varnothing$.

If the space $F \equiv E$ in Theorem 2 and $g$ therein is the identity mapping of $K$ onto itself, then we have

COROLLARY 2. Let $S$ be a nonempty convex subset of a separated locally convex space $E$ and $K$ a compact subset of $S$. Let $f: S \rightarrow E$ be a point closed, u.s.c. multifunction such that $f(x) \cap K$ is a nonempty convex set for each $x \in S$. Then $f$ has a fixed point in $K$.

It may be remarked that, when $S=K$, Corollary 1 provides a slightly weaker form of a result of Browder's theorem 4 [1]. Further, Theorem 2 is a generalization of Fan's theorem 2 [3] if the space $Y$ therein is locally convex.

\section{REFERENCES}

1. F. E. Browder, The fixed point theory of multi-valued mappings in topological vector spaces, Math. Ann. 177 (1968), 283-301. MR 37 \#4679.

2. K. Fan, Fixed-point and minimax theorems in locally convex topological linear spaces, Proc. Nat. Acad. Sci. U.S.A. 38 (1952), 121-126. MR 13, 858.

3. - A generalization of Tychonoff's fixed point theorem, Math. Ann. 142 (1960/61), 305-310. MR 24 \#A1120.

4. I. L. Glicksberg, A further generalization of the Kakutani fixed point theorem, with application to Nash equilibrium points, Proc. Amer. Math. Soc. 3 (1952), 170-174. MR 13, 764 . 
5. C. J. Himmelberg, Fixed points of compact multifunctions, J. Math. Anal. Appl. 38 (1972), 205-207.

6. S. Kakutani, A generalization of Brouwer's fixed point theorem, Duke Math. J. 8 (1941), 457-459. MR 3, 60.

7. A. Tychonoff, Ein fixpunktsatz, Math. Ann. 111 (1935), 767-776.

Department of Mathematics, University of Wyoming, Laramie, Wyoming 82070 